\title{
A PROPÓSITO DE LOS MODELOS DE MADUREZ DE GESTIÓN DEL CONOCIMIENTO*
}

\author{
LUIS MONTAÑEZ-CARRILLO** \& JENNY-PAOLA LIS-GUTIÉRREZ***** \\ UNIVERSIDAD CENTRAL
}

Recibido/Received/Recebido: 30/06/2016 - Aceptado/ Accepted/Aprovado: 30/09/2016

\begin{abstract}
Resumen
Este artículo tiene como propósito comparar los 24 Modelos de Madurez de Gestión del Conocimiento (MMGC), encontrados en la literatura académica de acceso abierto en: JSTOR, Emerald, Scopus, Science Direct, Taylor \& Francis, Scielo, Google Académico, EBSCO Business Source Premier y Engineering Village, entre los años 2001 y 2016. Los criterios de comparación utilizados correspondieron a los propuestos por Martínez (2015). Se identificó que el MMGC más apropiado para la realización del diagnóstico de madurez de GC es el Modelo general de madurez de GC (G-KMMM) de Teah, Pee \& Kankanhalli (2006).

Palabras clave: Gestión del conocimiento; Modelos de madurez; Niveles de madurez; Conocimiento.
\end{abstract}

\section{KNOWLEDGE MANAGEMENT MATURITY MODELS}

\begin{abstract}
The purpose of this article is to compare the 24 Knowledge Management Maturity Models (MMGC) found in the open access academic literature in: JSTOR, Emerald, Scopus, Science Direct, Taylor \& Francis, Scielo, Google Scholar, EBSCO Business Source Premier and Engineering Village between 2001 and 2016. The comparison criteria used corresponded to those proposed by Martínez (2015). It was identified that the most appropriate MMGC for the GC's maturity diagnosis execution is the Teah, Pee \& Kankanhalli's (2006) GC maturity general model.
\end{abstract}

Keywords: Knowledge management; Maturity models; Levels of maturity; Knowledge.

El artículo se deriva del trabajo de grado de la Maestría en Gestión del Conocimiento de la Universidad Central, titulado: "Diagnóstico de la madurez de la gestión del conocimiento en la Escuela de Ciencias Básicas, Tecnología e Ingeniería de la Universidad Nacional Abierta y a Distancia"

* Especialista en Pedagogía para el Desarrollo del Aprendizaje Autónomo, Estudiante de Maestría en Gestión de Organizaciones e Ingeniero de Sonido. Se desempeña actualmente como docente de la Escuela de Ciencias Básicas, Tecnología e Ingeniería de la Universidad Nacional Abierta y a Distancia. E-mail: luis.montanez@unad.edu.co

Candidata a doctora en Ingeniería, en el área de Industria y Organizaciones, Magíster en Análisis de problemas políticos, económicos e internacionales, Magíster en Sociedades contemporáneas comparadas Europa-América Latina, especialidad en Geografía y Planeación, Economista. Se desempeña actualmente como Directora del Centro de Investigaciones de la Escuela de Negocios de la Fundación Konrad Lorenz. E-mail: jenny.lis@konradlorenz.edu.co 


\title{
A PROPÓSITO DOS MODELOS DE MATURIDADE DE GERENCIAMENTO DO CONHECIMENTO
}

\begin{abstract}
Resumo
Este artigo tem como propósito comparar os 24 Modelos de Maturidade de Gerenciamento do Conhecimento (MMGC), encontrados na literatura acadêmica de acesso aberto em: JSTOR, Emerald, Scopus, Science Direct, Taylor \& Francis, Scielo, Google Acadêmico, EBSCO Business Source Premier e Engineering Village, entre os anos 2001 e 2016. Os critérios de comparação utilizados corresponderam aos propostos por Martínez (2015). Identificou-se que o MMGC mais apropriado para a realização do diagnóstico de maturidade de GC é o Modelo geral de maturidade de GC (G-KMMM) de Teah, Pee \& Kankanhalli (2006).

Palavras chave: Gerenciamento do conhecimento; Modelos de maturidade; Níveis de maturidade; Conhecimento.

Montañez-Carrillo, L. \& Lis-Gutiérrez, J. (2017) A propósito de los Modelos de Madurez de Gestión del Conocimiento. En: Revista de la Facultad de Ciencias Económica: Investigación y Reflexión. rev.fac.cienc.econ, XXV (2), DOI: https://doi.org/10.18359/rfce.3069
\end{abstract}

JEL: M10, M19, 032, D83, D89.

\section{Introducción}

A partir de los cambios provocados por la emergencia de la sociedad de la información y el conocimiento se ha redefinido el papel de este último en las instituciones al identificarlo como un factor creador de riqueza en las organizaciones para su éxito y perdurabilidad (Heinzen, 2015). No basta, sin embargo, con gestionarlo de manera adecuada, se requiere ir más allá y analizar los diferentes estadios en los cuales una organización puede crear, capturar, transformar y usar el conocimiento orientado al desarrollo organizacional y la creación de ventajas competitivas (Olarte-Reyes \& Lis-Gutiérrez, 2015).

En este sentido, el presente documento pretende responder ¿cuáles son los Modelos de Gestión del Conocimiento (MMGC) propuestos por la literatura entre 2001 y 2016 y cuál de ellos es el más apropiado para realizar diagnósticos de madurez de gestión del conocimiento (GC)? Los MMGC se comprenderán como mecanismos que permiten medir y comparar las iniciativas que emprende una organización en aras de gestionar el conocimiento que en ella se crea. Los modelos permiten establecer el estado actual en el que se encuentra una organización en materia de GC y definir una ruta para mejorar el estado actual. En esta medida se establecen como guías para la implementación de la GC en etapas y contemplan el tránsito gradual de un estado inicial hasta uno superior u óptimo.

Para ello, en este trabajo se realizó una revisión de la literatura académica que propusiese, analizara o utilizara los modelos de madurez de gestión del conocimiento (MMGC), entre 2001 y $2016^{1}$ y que estuviera disponible en JSTOR, Emerald, Scopus, Science Direct, Taylor \& Francis, Scielo, Google Académico, EBSCO Business Source Premier y Engineering Village. Esto con el fin de elaborar un balance tanto de los trabajos publicados en revistas de circulación restringida (por suscripción) y los textos disponibles para el público en general, vía open access.

Posteriormente, considerando los criterios de comparación propuestos por Martínez (2015), se llevó a cabo la calificación de cada uno de ellos en los mode-

\footnotetext{
El año de inicio del período seleccionado corresponde a la fecha en la cual comienzan a aparecer los trabajos sobre madurez de gestión del conocimiento.
} 
los encontrados. De esta manera se identificaron las fortalezas y debilidades de cada uno de los MMGC encontrados y se determinó cuál es el más robusto.

Con el fin de cumplir con el objetivo trazado, el artículo se estructura en cuatro partes. En la primera se realiza la presentación de la metodología; en la segunda una revisión de la literatura; en la tercera un análisis y discusión de la literatura, proponiendo aspectos que permitan comparar y elegir entre los diferentes MMGC. Por último, se formulan las consideraciones finales.

\section{Metodología}

Para este trabajo se estableció que el tipo de investigación es descriptiva ya que se busca especificar las propiedades o características del fenómeno que se quiere analizar. Para la elaboración del estado del arte sobre MMGC se realizó una revisión sistemática de fuentes secundarias, es decir, una revisión de la literatura en el ámbito académico en materia de modelos para medir la madurez de GC, a través de búsquedas en bases de datos académicas tanto estructuradas como no estructuradas, de trabajos de investigación y artículos sobre el tema objeto de estudio, tales como Sciencedirect, Scopus, Engineering Village, IEEE, EBSCO, JSTOR, Emerald Insight, Google Académico, Taylor \& Francis y Scielo. Los textos pertenecen a diferentes regiones geográficas: América del Norte, América Latina, Europa, Asia y África.

Los trabajos de investigación encontrados se enfocan específicamente en el tema de madurez de gestión de conocimiento (GC) y fueron desarrollados entre 2001 y 2016, por lo cual es posible indicar que los estudios sobre la madurez de GC son relativamente recientes. Una vez culminada la revisión de literatura en materia de modelos para medir la madurez de GC, se identificaron 24 modelos empleados en el período.

Después de realizar la revisión de literatura, se llevó a cabo un análisis comparativo con los 24 modelos encontrados, en aras de identificar el más apropiado para la realización del diagnósticos de madurez de GC. En la elaboración del análisis comparativo se emplearon una serie de criterios de comparación que se plantearon tomando como referente los criterios propuestos por Martínez (2015).

Dichos criterios fueron:

- Niveles de madurez. Corresponde al número de niveles de madurez que el modelo emplea para indicar los estados de las capacidades y prácticas en materia de GC (Arias-Pérez et al., 2016)

- Áreas o elementos clave. Agrupan las capacidades y prácticas de GC que desarrolla una organización (Arias-Pérez et al., 2016)

- Instrumento. El modelo cuenta con un cuestionario o encuesta para la medición de la madurez de GC con preguntas de selección múltiple.

- Brevedad del instrumento. El instrumento es sencillo, no toma demasiado tiempo en su aplicación y sus preguntas no son demasiado técnicas.

- Idioma. Lengua en la cual se encuentra el instrumento del modelo para medir la madurez de GC.

- Complejidad. El modelo es flexible, robusto y sencillo para ser aplicado en la medición de la madurez de GC.

- Amplio y adaptable. El modelo posee una amplia documentación y se puede adaptar a diferentes contextos.

- Libre uso. Se refiere a que el modelo se pueda emplear libremente en su totalidad a partir de la documentación existente.

Una vez encontradas las debilidades y fortalezas de los 24 modelos para medir la madurez de GC a través del análisis comparativo, se aplicó una evaluación de orden cuantitativo, asignando una calificación de 0 a $1^{2}$ a cada criterio de acuerdo con las escalas que se presentan a continuación:

Este rango se definió dado que hay variables binarias (existencia $=1$ ó ausencia $=0$ ). Para el caso de las variables con una opción intermedia, se usa el valor de 0,5. 
- $\quad$ Criterio de niveles de madurez

\begin{tabular}{|c|c|c|c|}
\hline & No define & Menos de 4 niveles & Igual o más de 4 niveles \\
\hline Calificación & 0 & 0,5 & 1 \\
\hline
\end{tabular}

- Criterio áreas o elementos clave

\begin{tabular}{|c|c|c|c|}
\hline & No define & Entre 2 y $\mathbf{5}$ niveles & Más de $\mathbf{5}$ niveles \\
\hline Calificación & 0 & 0,5 & 1 \\
\hline
\end{tabular}

- Criterio instrumento (binaria)

\begin{tabular}{|c|c|c|}
\hline & No tiene instrumento & Si tiene instrumento \\
\hline Calificación & 0 & 1 \\
\hline
\end{tabular}

- Criterio brevedad del instrumento

\begin{tabular}{|c|c|c|c|}
\hline & $\begin{array}{c}\text { Baja } \\
\text { (> a 100 preguntas) }\end{array}$ & $\begin{array}{c}\text { Media } \\
\text { (50-100 preguntas) }\end{array}$ & $\begin{array}{c}\text { Alta } \\
\text { (< a 50 preguntas) }\end{array}$ \\
\hline Calificación & 0 & 0,5 & 1 \\
\hline
\end{tabular}

- $\quad$ Criterio idioma (binaria)

\begin{tabular}{|c|c|c|c|}
\hline & Otro idioma & Inglés & Español \\
\hline Calificación & 0 & 0,5 & 1 \\
\hline
\end{tabular}

- $\quad$ Criterio complejidad

\begin{tabular}{|c|c|c|c|}
\hline & $\begin{array}{c}\text { Alta } \\
\text { (Poco flexibles y poco } \\
\text { prácticos de aplicar) }\end{array}$ & $\begin{array}{c}\text { Media } \\
\text { (Flexibles y medianamen- } \\
\text { te prácticos de aplicar) }\end{array}$ & $\begin{array}{c}\text { Baja } \\
\text { (Flexibles y prácticos de } \\
\text { aplicar) }\end{array}$ \\
\hline Calificación & 0 & 0,5 & 1 \\
\hline
\end{tabular}

- Criterio amplio y adaptable (binaria)

\begin{tabular}{|c|c|c|}
\hline & No & Si \\
\hline Calificación & 0 & 1 \\
\hline
\end{tabular}

- $\quad$ Criterio libre uso (binaria)

\begin{tabular}{|c|c|c|}
\hline & No & Si \\
\hline Calificación & 0 & 1 \\
\hline
\end{tabular}




\section{Revisión de la literatura}

De acuerdo con Prieto, Meneses, \& Vega (2015), los modelos de madurez brindan la posibilidad a las organizaciones de medir y comparar sus iniciativas en diferentes ámbitos, indican el estado actual en el que se encuentra la organización y describen el camino hacia dónde debe moverse desde su ubicación actual. Estos autores también mencionan que existen diversos modelos de madurez para una gran variedad de ámbitos (Gestión de Proyectos, Inteligencia de Negocios, mejores prácticas para el proceso de desarrollo de software, entre otros) y que algunos han surgido de la academia y otros de organizaciones tanto privadas como públicas.

Ahora bien, específicamente los MMGC se basan en la necesidad de tener una hoja de ruta clara para cualquier organización que busca implementar la GC. Brindan una visión clara con una descripción del camino a seguir y proporcionan una comprensión común de los términos y elementos involucrados en la GC (Klimko, 2001).

Desde el punto de vista de Arias-Pérez et al. (2016) en las últimas dos décadas el conocimiento ha empezado a tener más importancia en el ámbito empresarial, debido al hecho que es el recurso que mayor incidencia tiene sobre el desempeño organizacional y la creación de ventajas competitivas. De acuerdo con dichos autores, a principios del siglo XXI se comienza a articular la GC con los modelos de madurez que surgieron de la ingeniería de software, $\mathrm{y}$ de este modo se estructuraron las primeras guías para la implementación de la GC en etapas, que contemplan el tránsito gradual de un estado inicial hasta uno óptimo. La estructura básica de los MMGC desde la perspectiva de estos Arias-Perez et al. (2016) es la siguiente:

- Áreas clave. Agrupan las capacidades y prácticas de GC que desarrolla una organización, de acuerdo con la articulación que tengan con elementos como la tecnología, la cultura y los procesos, entre otros.

- Escala de madurez. Regularmente los modelos de este tipo constan de niveles que indican los distintos estados de las capacidades y prácticas en materia de GC, partiendo de un nivel principiante hasta llegar a un máximo de desarrollo y consolidación de la organización.

\subsection{Revisión internacional distinta a América Latina}

En el ámbito internacional, principalmente en las regiones de América del Norte, Europa, Asia y Áfri$\mathrm{ca}$, se encontraron trabajos de investigación que se caracterizan por proponer el diseño y aplicación de modelos y metodologías de madurez de GC.

Antes de abordar directamente los MMGC es necesario explorar el modelo denominado "Capability Maturity Model for Software (CMM)", el cual fue el primer modelo de madurez y fue el referente más importante para la construcción de los MMGC. Desarrollado por Paulk, Curtis, Chrissis \& Webwe (1993) del Software Engineering Institute de Carnegie Mellon University, describe un camino de mejora evolutiva de un proceso inmaduro a un proceso maduro y disciplinado.

El CMM propone cinco etapas: 1-inicial (procesos caóticos), 2-repetible (procesos que se planearon y ejecutaron con una política), 3-definido (procesos estandarizados), 4-gestionado (gestión del rendimiento de los procesos) y 5-optimizado (mejora continua de los procesos) (Kuriakose et al., 2010). El modelo también define las características que marcan la evolución de dichos procesos y las áreas clave en las que se debe implementar un conjunto de prácticas o enfocarse los cambios con el objeto de definir los mejoramientos (Durango et al., 2013).

Paulzen, Doumi, Perc \& Cereijo-Roibas (2002) proponen el modelo Knowledge Process Quality Model (KPQM), que se basa en las ideas de gestión de calidad e ingeniería de procesos. Este modelo ayuda a las organizaciones a evaluar y mejorar sus estructuras de GC para controlar los procesos de conocimiento, así se apoya el aprendizaje sistemático de GC.

Por su parte, Kulkarni \& St Louis (2003) desarrollaron un instrumento denominado Autoevaluación 
Organizacional de la Madurez de la GC, para la autoevaluación de la madurez de GC. El instrumento define 5 niveles de madurez que se derivan del modelo CMM.

Más tarde, Snyman \& Kruger (2005) desarrollaron un modelo de madurez de GC. Los autores indican que la madurez de la GC debe derivarse de la capacidad para conducir al crecimiento de la organización y la rentabilidad. Sin embargo, se argumenta que las organizaciones tienen que progresar a un punto en que sean capaces de gestionar las tecnologías de la información y comunicación (TIC) y el conocimiento de forma simultánea.

Posteriormente, Feng (2006) propuso un modelo de madurez de GC, basado en el concepto de mejora continua de procesos y el CMM. Este modelo puede ser empleado para describir como las organizaciones soportan las prácticas de cada nivel de madurez y brinda caminos para lograr un alto grado de madurez.

Harris (2006) planteó un MMGC que le permite a las organizaciones determinar el nivel actual de madurez y establecer dónde se deberían encontrar, cómo deben invertir en la comprensión de la GC y cómo deben plantear acciones para avanzar al siguiente nivel.

Así mismo, Teah, Pee \& Kankanhalli (2006) proponen un modelo general de madurez de GC (GKMMM) que se centra en la evaluación de la madurez de las personas, los procesos y los aspectos tecnológicos en el desarrollo de los conocimientos en las organizaciones. Los autores también desarrollaron una herramienta de evaluación para facilitar la aplicación práctica. Este modelo puede servir como una herramienta útil que describe y orienta los esfuerzos de implementación de GC proporcionando una descripción clara de la situación actual y las indicaciones del camino a seguir. El modelo G-KMMM adaptó los cinco niveles de madurez del CMM: inicial, conciencia, definido, gestionado y de optimización.

El modelo general de madurez de GC tiene una estructura por etapas y tres componentes principales denominados: (i) niveles de madurez, (ii) áreas de procesos clave (organización - personas, procesos y tecnología) y (iii) características comunes (Durango et al., 2013).

Igualmente, Joslin (2007) propuso un MMGC denominado "The Support Center Maturity Model". El modelo examina las características tales como: personas, procesos, tecnología y visión. También proporciona orientación a las dependencias relacionadas con el soporte técnico en la medida que evolucionan y se vuelven más valiosas para sus empresas. El modelo define cuatro fases de madurez: reactiva, proactiva, centrada en el cliente y centrada en la empresa.

Gaál, Szabó, Kovács, Obermayer-Kovács \& Csepregi (2008) formularon el modelo "Knowledge management profile maturity model". Este define áreas dentro de las cuales las organizaciones pueden desarrollar la práctica de GC: conciencia, almacenamiento, participación, tecnología, información, comunidad, infraestructura.

Por su parte, Hsieh, Lin \& Lin (2009) desarrollaron el MMGC "knowledge navigator model (KNM)" que clasifica los niveles de madurez en 5 escenarios: caótico de conocimiento, consciente de conocimiento, de GC, de GC avanzado y de GC integrado. El marco de evaluación del KNM propone tres aspectos: tres objetos de gestión estratégicos: cultura, proceso de la GC y Tecnologías de la Información. También propone 68 actividades de GC y 16 áreas clave.

Arling \& Chun (2011) presentaron el diseño de un marco que tiene como fin evaluar la capacidad de un sistema de GC para facilitar la creación de nuevo conocimiento. Los autores establecieron que la generación de nuevo conocimiento se apoya mejor a través de los sistemas de GC que tienen un alto índice de madurez, y que a su vez incluyen los cuatro modos de creación de conocimiento: combinación, externalización, socialización e internalización.

En esta misma óptica, Kuriakose, Raj, Satya Murty \& Swaminathan (2011) presentaron un modelo que combina las fortalezas y elimina las deficiencias de los modelos existentes, con flexibilidad, adaptabili- 
dad y facilidad de uso práctico. El modelo incluye un concepto de Indicador de madurez clave, lo cual hace que sea más flexible.

Wibowo \& Waluyo (2015) elaboraron un estudio cuyo propósito era identificar la madurez de GC en empresas de construcción en Indonesia, para lo cual aplicaron cuestionarios a las grandes empresas que implementan actividades de GC. El estudio propo- nía 4 niveles de madurez de GC: 1) inicial, 2) practicado 3) gestionado 4) continuamente mejorado. Los resultados establecieron que 29 constructoras tenían el nivel 2 (practicado), 20 el nivel 3 (gestionado) y 5 el nivel 4 (continuamente mejorado).

En la tabla 1 se presenta un resumen y las principales características de los modelos para medir la madurez de GC encontrados en la revisión internacional.

Tabla 1. Revisión internacional de MMGC

\begin{tabular}{|c|c|c|c|}
\hline $\begin{array}{c}\text { Denominación del modelo } \\
\text { o estudio }\end{array}$ & Autor(es) & Niveles de madurez de GC & Áreas o elementos clave \\
\hline $\begin{array}{l}\text { Capability Maturity Model for } \\
\text { Software (CMM) }\end{array}$ & $\begin{array}{l}\text { Paulk, Curtis, Chrissis \& We- } \\
\text { bwe (1993) }\end{array}$ & $\begin{array}{l}5 \text { etapas: Inicial, repetible, definido, } \\
\text { gestionado y optimizado }\end{array}$ & $\begin{array}{l}\text { Nivel } 2 \text { - repetible } \\
\text { Nivel } 3 \text { - definido. } \\
\text { Nivel } 4 \text { - gestionado } \\
\text { Nivel } 5 \text { - optimizado }\end{array}$ \\
\hline $\begin{array}{l}\text { Knowledge Process Quality } \\
\text { Model (KPQM) }\end{array}$ & $\begin{array}{l}\text { Paulzen, Doumi, Perc \& Cerei- } \\
\text { jo-Roibas (2002) }\end{array}$ & $\begin{array}{l}5 \text { etapas: inicial, consciente, estable- } \\
\text { cido, gestionado cuantitativamente, } \\
\text { optimización }\end{array}$ & $\begin{array}{l}\text { 1) Etapa de madurez } \\
\text { 2) Actividad de conocimiento } \\
\text { 3) Área de Gestión } \\
\text { 4) Estructura de evaluación }\end{array}$ \\
\hline $\begin{array}{l}\text { Organizational Self Assessment } \\
\text { of Knowledge Management } \\
\text { Maturity }\end{array}$ & Kulkarni \& St Louis (2003) & $\begin{array}{l}5 \text { niveles: posible, alentado, habilitado / } \\
\text { practicado, gestionado, continuamente } \\
\text { mejorado }\end{array}$ & $\begin{array}{l}\text { Lecciones aprendidas } \\
\text { Experticia } \\
\text { Datos } \\
\text { Conocimiento estructurado }\end{array}$ \\
\hline $\begin{array}{l}\text { Strategic knowledge management } \\
\text { maturity model }\end{array}$ & Snyman \& Kruger (2005) & $\begin{array}{l}4 \text { niveles: inicial, consciente, gestionar } \\
\text { y optimizar }\end{array}$ & $\begin{array}{l}\text { Directores } \\
\text { Políticas } \\
\text { Estrategia de conocimiento } \\
\text { Estrategias de GC }\end{array}$ \\
\hline $\begin{array}{l}\text { A Knowledge Management } \\
\text { Maturity Model and Application }\end{array}$ & Feng (2006) & $\begin{array}{l}5 \text { niveles: nivel de inicialización, nivel } \\
\text { de iteración, nivel de definición, nivel } \\
\text { gerencial, nivel de optimización }\end{array}$ & $\begin{array}{l}\text { Cultura } \\
\text { Estructura } \\
\text { Ciencia y Tecnología }\end{array}$ \\
\hline $\begin{array}{l}\text { Gartner: A Knowledge } \\
\text { Management Maturity Model } \\
\text { Explains Where You ' re Going } \\
\text { and How to Get There }\end{array}$ & Harris (2006) & $\begin{array}{l}6 \text { niveles: no existente, inicial, repeti- } \\
\text { ble, definido, gestionado, optimizado }\end{array}$ & $\begin{array}{l}\text { Estrategia e intención } \\
\text { Infraestructura } \\
\text { Fuentes del conocimiento y uso }\end{array}$ \\
\hline $\begin{array}{l}\text { Modelo general de madurez de } \\
\text { GC (G-KMMM) }\end{array}$ & $\begin{array}{l}\text { Teah, Pee \& Kankanhalli } \\
(2006)\end{array}$ & $\begin{array}{l}\text { Adapta los cinco niveles de madurez } \\
\text { del CMM de Paulk, et al. (1993): Inicial, } \\
\text { conciencia, definido, gestionado / es- } \\
\text { tablecido y de optimización / compartir }\end{array}$ & $\begin{array}{l}\text { Personas / organización } \\
\text { Procesos } \\
\text { Tecnología }\end{array}$ \\
\hline
\end{tabular}




\begin{tabular}{|c|c|c|c|}
\hline $\begin{array}{c}\text { Denominación del modelo } \\
\text { o estudio }\end{array}$ & Autor(es) & Niveles de madurez de GC & Áreas o elementos clave \\
\hline $\begin{array}{l}\text { The Support Center Maturity } \\
\text { Model }\end{array}$ & Joslin (2007) & $\begin{array}{l}4 \text { fases de madurez: reactiva, proacti- } \\
\text { va, centrada en el cliente y centrada en } \\
\text { la empresa }\end{array}$ & $\begin{array}{l}\text { Personas } \\
\text { Procesos } \\
\text { Tecnología } \\
\text { Visión }\end{array}$ \\
\hline $\begin{array}{l}\text { Knowledge management profile } \\
\text { maturity model }\end{array}$ & $\begin{array}{l}\text { Gaál, Szabó, Kovács, Ober- } \\
\text { mayer-Kovács \& Csepregi } \\
(2008)\end{array}$ & No define & $\begin{array}{l}\text { Conciencia, almacenamiento, partici- } \\
\text { pación, tecnología, información, co- } \\
\text { munidad, infraestructura }\end{array}$ \\
\hline $\begin{array}{l}\text { knowledge navigator model } \\
\text { (KNM) }\end{array}$ & Hsieh, Lin \& Lin (2009) & $\begin{array}{l}5 \text { etapas: escenario caótico de conoci- } \\
\text { miento, escenario consciente de cono- } \\
\text { cimiento, escenario de GC, escenario } \\
\text { de GC avanzado y escenario de GC } \\
\text { integrado }\end{array}$ & $\begin{array}{l}\text { Cultura } \\
\text { Proceso de la GC } \\
\text { Tecnologías de la Información }\end{array}$ \\
\hline $\begin{array}{l}\text { Facilitating new knowledge } \\
\text { creation and obtaining KM } \\
\text { maturity }\end{array}$ & Arling \& Chun (2011) & 3 niveles: bajo, medio, alto & $\begin{array}{l}\text { Combinación, externalización, sociali- } \\
\text { zación e internalización. }\end{array}$ \\
\hline $\begin{array}{l}\text { Knowledge Management Maturity } \\
\text { Model: An Engineering Approach }\end{array}$ & $\begin{array}{l}\text { Kuriakose, Raj, Satya Murty \& } \\
\text { Swaminathan (2011) }\end{array}$ & $\begin{array}{l}6 \text { niveles: por defecto, inicial, desarro- } \\
\text { llo cualitativo, desarrollo cuantitativo, } \\
\text { madurez, madurez organizacional }\end{array}$ & $\begin{array}{l}\text { Personas } \\
\text { Procesos } \\
\text { Tecnología } \\
\text { Conocimiento } \\
\text { Retorno sobre la inversión }\end{array}$ \\
\hline $\begin{array}{l}\text { Knowledge Management Maturity } \\
\text { in Construction Companies }\end{array}$ & Wibowo \& Waluyo (2015) & $\begin{array}{l}4 \text { niveles: 1) Inicial, 2) practicado } 3 \text { ) } \\
\text { gestgionado 4) continuamente mejo- } \\
\text { rado }\end{array}$ & Medición general de la madurez de GC \\
\hline
\end{tabular}

Fuente: elaboración propia a partir de la revisión de literatura.

\subsection{Revisión en América Latina}

En países como Chile, Brasil, México y Colombia se encontraron trabajos de investigación que se caracterizan por proponer MMGC y estudios de aplicación de algunos modelos de madurez (existentes) en empresas de dichos países. En el caso específico de Colombia se encontró que los trabajos se concentran principalmente en diagnósticos sobre la madurez de GC en empresas importantes y se realizan análisis comparativos de modelos de madurez.

Para iniciar, González et al. (2004) desarrollaron una metodología para diagnosticar el estado de la GC en una importante empresa de Barranquilla. Dicha metodología surgió del análisis y sintesis de los principales exponentes de la GC, de las mejores prácticas encontradas, del modelo de capital intelectual y de la espiral del conocimiento de Nonaka \&
Takeuchi (1995). Con la metodología propuesta fue posible determinar seis variables macro claves: identificación del conocimiento, proceso de transmisión del conocimiento, medios y tecnologías, toma de decisiones, cultura organizacional y competitividad, las cuales permitieron evaluar la GC de la empresa en una escala de cero a cinco.

González, Joaquí \& Collazos (2009) presentaron el modelo "Karagabi KM Model" que sirve como guía de referencia para la construcción de organizaciones orientadas al conocimiento. El modelo propone tres áreas clave: metodología de intervención, librería de modelos de conocimiento y base de conocimiento de experiencias.

Más tarde, Briceño \& Bernal (2010) realizaron un estudio de caso donde evidencian que existe una gran coincidencia entre el concepto y el enfoque 
que tienen las organizaciones sobre GC, pero que hay un contraste en la importancia que cada organización da a cada una de las variables que componen dicha gestión.

Oliveira, Pedron, Romão \& Becker (2011) desarrollaron el modelo KM3. Este modelo consta de cinco etapas: Falta conciencia (2 factores), Planificación (15 factores), Iniciación (20 factores), Desarrollo (24 factores) y la integración (24 factores). Adicionalmente establece tres (3) áreas clave: cultura organizacional, soporte de alta administración y estructura organizacional.

Por su parte, Romero \& Pascual (2011) propusieron un modelo exploratorio que se basa en una relación causa - efecto que a su vez resulta de la vinculación entre las TI/SI (planificación estratégica) la cultura y el capital humano con la GC en el marco de un modelo de madurez.

Arias (2012) presentó un estudio para determinar asociaciones entre la madurez de la GC (áreas de personas y organización, e interpretación), con el desempeño innovador (resultados en innovación de producto, proceso, organizacional y mercadotecnia). El estudio concluyó que las áreas de personas y organización, e interpretación no tienen mayor injerencia sobre la innovación de producto, sin embargo, las innovaciones más intangibles (procesos, organizacional, y mercadotecnia) evidencian asociaciones con la GC.

Así mismo, Serna (2012) elaboró un artículo que constituye una extensa documentación sobre el tema de GC, centrado en la revisión, análisis y presentación de un estudio de perspectiva interpretativista que describe un modelo de madurez para la GC operativo. Por otra parte, Uribe (2013) analizó el estado de la GC en algunas Instituciones de Educación Superior de la ciudad de Medellín. La investigación surgió de un análisis situacional (caracterización de la información obtenida por fuentes primarias). El autor encontró que existe una gran diversidad conceptual del tema por parte de los responsables del área en cada institución y evidenció grandes vacíos en las IES estudiadas, donde la GC se queda en el plano de la transferencia.
Durango \& Pérez (2013) buscaron construir un MMGC en grandes empresas de la ciudad de Medellin, desde las perspectivas funcionalista e interpretativa; evaluaron la madurez de las áreas clave de tecnología y procesos de GC en dichas empresas. El estudio concluyó que el modelo de madurez de la GC propuesto era sistemático y apropiado para la evaluación de las áreas clave procesos y tecnología, mostrando los resultados de la medición del nivel de madurez de manera global e independiente para cada una de las áreas clave.

Ese mismo año, Durango et al., (2013) propusieron una metodología para evaluar la madurez de GC en 13 organizaciones colombianas, a partir de las perspectivas epistemológicas funcionalista $e$ interpretativa, usando de manera exploratoria el análisis clúster para la clasificación de madurez, a través de cuatro dimensiones o áreas clave de la GC. Con la metodología fue posible la realización de la medición del nivel de madurez de manera global por empresa y para cada una de las áreas clave, así como la comparación de las empresas según los cinco niveles de la escala de madurez: inicial, conciencia, definido, gestionado y optimizado.

Posteriormente, Acuña, Gómez, \& Pintor (2014) elaboraron una investigación que evaluaba el nivel de madurez de GC y la gestión de procesos en 2 empresas que empleaban la plataforma TALENTUM (plataforma que proporciona capacidad ilimitada de almacenamiento y tráfico de usuarios). El estudio concluyó que TALENTUM era un instrumento que fomenta la madurez en los procesos de GC y el desarrollo de capacidades en las empresas. La metodología desarrollada por los autores propuso 5 niveles de madurez de GC (I, II, III, IV y V) y seis (6) áreas clave: identificar el conocimiento, obtener el conocimiento, almacenar el conocimiento, desarrollar competencias, compartir el conocimiento y utilizar el conocimiento.

Follador (2015) desarrolló una investigación cuyo propósito era averiguar como la GC en un entorno de prueba de vuelo de la Fuerza Aérea Brasileña se vio afectada por el establecimiento de una estructura de gestión de la ciencia y tecnología. Los resultados arrojaron que después del cambio de estructura 
fue posible observar un cierto desarrollo de los procesos de GC, sin embargo, no produjo un impacto significativo en el nivel de madurez de GC. La metodología propuesta por el autor para la medición del nivel de madurez de GC constaba de 3 niveles: avanzado, intermedio e inicial, adicionalmente estableció las áreas clave de personas, cultura, procesos y tecnología.

Arias-Pérez \& Durango-Yepes (2015) desarrollaron un estudio para analizar la madurez de GC de grandes empresas que habían explorado las prácticas de GC en la ciudad de Medellín. Los autores elaboraron un modelo de madurez que integraba las perspectivas de GC: funcionalista e interpretativa. El modelo estableció 4 áreas clave: organización y personas, procesos, tecnología e interpretación de la GC, y definió los rangos de los niveles de madurez de GC: inicial, consciencia, definido, gestionado y optimizado. Dentro de los resultados, los autores encontraron que únicamente dos empresas superaron el nivel definido y que los mejores resultados se lograron en el área clave de tecnología.

Arias \& Tavera (2015) realizaron un artículo cuyo objetivo fue explorar la relación entre la madurez de GC y las innovaciones de producto y marketing en empresas líderes en I+D. Los resultados indicaron que la recopilación del conocimiento era la variable que presentaba la asociación más significativa con ambos tipos de innovación. Como conclusión se resalta que es evidente la importancia de las variables de GC de corte cultural con la innovación de producto, mientras que los aspectos metodológicos tienen un mayor impacto sobre la innovación de marketing.

Finalmente, Arias-Pérez et al., (2016) construyeron $y$ aplicaron un MMGC en una multinacional de alimentos. El modelo creado por los autores, estableció cuatro (4) áreas clave: estrategia, cultura, procesos y tecnología, y una escala de cinco (5) niveles de madurez: 1) inicial, 2) exploratorio, 3) usado, 4) gestionado e 5) innovación. Los autores aplicaron un instrumento a 3.000 empleados de catorce (14) unidades de negocio, encontrando que dentro de la multinacional de alimentos todas las áreas clave se encontraban en el nivel Gestionado (4), no obstante, el análisis por cada área clave presentó que la organización tuvo mayor desarrollo en Cultura y Estrategia.

Un resumen de los modelos para medir la madurez de GC encontrados en la revisión de literatura en América Latina y sus principales características se presentan en la tabla 2. 
Tabla 2. Revisión en América Latina de MMGC.

\begin{tabular}{|c|c|c|c|}
\hline Denominación del modelo o estudio & Autor(es) & Niveles de madurez de GC & Áreas o elementos clave \\
\hline $\begin{array}{l}\text { Diagnóstico de la gestión de conocimiento en una } \\
\text { empresa grande de Barranquilla (Colombia) }\end{array}$ & $\begin{array}{l}\text { González, Castro \& } \\
\text { Roncallo (2004) }\end{array}$ & $\begin{array}{l}5 \text { niveles: bajo, medio bajo, me- } \\
\text { dio, medio alto y alto }\end{array}$ & $\begin{array}{l}\text { Identificación del conocimiento, Pro- } \\
\text { ceso de transmisión del conocimien- } \\
\text { to, Medios y tecnologías, Toma de } \\
\text { decisiones, Cultura organizacional y } \\
\text { Competitividad }\end{array}$ \\
\hline Karagabi KM Model & $\begin{array}{l}\text { González, Joaquí \& } \\
\text { Collazos (2009) }\end{array}$ & No define & $\begin{array}{l}\text { Metodología de intervención, librería } \\
\text { de modelos de conocimiento, y la } \\
\text { base de conocimiento de experien- } \\
\text { cias }\end{array}$ \\
\hline $\begin{array}{l}\text { Estudios de caso sobre la GC en cuatro organi- } \\
\text { zaciones colombianas líderes en penetración de } \\
\text { mercado }\end{array}$ & $\begin{array}{l}\text { Briceño \& Bernal } \\
(2010)\end{array}$ & No establece & $\begin{array}{l}\text { Identificación del conocimiento, pro- } \\
\text { ceso de transmisión del conocimien- } \\
\text { to, medios y tecnologías, toma de } \\
\text { decisiones, cultura organizacional y } \\
\text { competitividad }\end{array}$ \\
\hline KM3 & $\begin{array}{l}\text { Oliveira, Pedron, } \\
\text { Romão \& Becker } \\
(2011)\end{array}$ & $\begin{array}{l}\text { Falta conciencia, Planificación, } \\
\text { Iniciación, Desarrollo e integra- } \\
\text { ción }\end{array}$ & $\begin{array}{l}\text { Cultura organizacional, soporte de } \\
\text { alta administración y estructura or- } \\
\text { ganizacional }\end{array}$ \\
\hline $\begin{array}{l}\text { Análisis de madurez de la gestión del capital in- } \\
\text { telectual en la pequeña y mediana empresa lati- } \\
\text { noamericana }\end{array}$ & $\begin{array}{l}\text { Romero \& Pascual } \\
(2011)\end{array}$ & $\begin{array}{l}4 \text { niveles: codificación, abstrac- } \\
\text { ción, difusión y uso }\end{array}$ & $\begin{array}{l}\text { Cultura organizacional, tecnología de } \\
\text { información, capital humano. }\end{array}$ \\
\hline $\begin{array}{l}\text { La GC en Instituciones de Educación Superior } \\
\text { (IES) de Medellín }\end{array}$ & Uribe (2013) & No establece & $\begin{array}{l}\text { Modelos pedagógicos, concepción } \\
\text { teórica de la GC, aplicación de la GC, } \\
\text { evidencias de la GC, dificultades para } \\
\text { la GC }\end{array}$ \\
\hline $\begin{array}{l}\text { Análisis comparativo de los modelos de madu- } \\
\text { rez de la GC en grandes empresas de Medellín- } \\
\text { Colombia }\end{array}$ & $\begin{array}{l}\text { Durango \& Pérez } \\
\text { (2013) }\end{array}$ & $\begin{array}{l}5 \text { niveles: Inicial, conciencia, defi- } \\
\text { nido, gestionado/establecido y de } \\
\text { optimización/compartir }\end{array}$ & Tecnología y procesos \\
\hline $\begin{array}{l}\text { Metodología para evaluar la madurez de la GC en } \\
\text { algunas grandes empresas colombianas }\end{array}$ & $\begin{array}{l}\text { Durango, Quintero \& } \\
\text { Ruiz (2013) }\end{array}$ & $\begin{array}{l}5 \text { niveles: Inicial, conciencia, defi- } \\
\text { nido, gestionado/establecido y de } \\
\text { optimización/compartir }\end{array}$ & 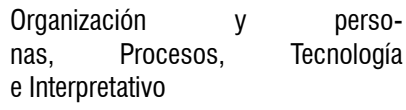 \\
\hline $\begin{array}{l}\text { Madurez de la GC y la gestión de procesos en } \\
\text { empresas que utilizan la plataforma virtual TA- } \\
\text { LENTUM }\end{array}$ & $\begin{array}{l}\text { Acuña, Gómez, \& } \\
\text { Pintor (2014) }\end{array}$ & 5 niveles: I, II, III, IV y V & $\begin{array}{l}\text { Identificar el conocimiento, obtener } \\
\text { el conocimiento, almacenar el cono- } \\
\text { cimiento, desarrollar competencias, } \\
\text { compartir el conocimiento y utilizar el } \\
\text { conocimiento }\end{array}$ \\
\hline $\begin{array}{l}\text { Knowledge Management Maturity Level in a Brazi- } \\
\text { lian Air Force Flight Test Environment }\end{array}$ & Follador (2015) & $\begin{array}{l}3 \text { niveles: avanzado, intermedio } \\
\text { e inicial }\end{array}$ & $\begin{array}{l}\text { Personas, cultura, procesos y tecno- } \\
\text { logía }\end{array}$ \\
\hline $\begin{array}{l}\text { Exploring knowledge management maturity from } \\
\text { funcionalist and interpretivist perspectives*. }\end{array}$ & $\begin{array}{l}\text { Arias-Pérez \& Duran- } \\
\text { go-Yepes (2015) }\end{array}$ & $\begin{array}{l}5 \text { niveles: inicial, consciencia, } \\
\text { definido, gestionado y optimizado }\end{array}$ & $\begin{array}{l}\text { Organización y personas, procesos, } \\
\text { tecnología e interpretación de la GC }\end{array}$ \\
\hline $\begin{array}{l}\text { Construcción De Un MMGC Para Una Multinacio- } \\
\text { nal De Alimentos De Una Economía Emergente. }\end{array}$ & $\begin{array}{l}\text { Arias-Pérez, } \\
\text { Tavera-Mesías \& } \\
\text { Castaño-Serna } \\
\text { (2016) }\end{array}$ & $\begin{array}{l}5 \text { niveles de madurez: 1) Inicial, } \\
\text { 2) Exploratorio, 3) Usado, 4) Ges- } \\
\text { tionado e 5) Innovación }\end{array}$ & $\begin{array}{l}\text { Estrategia, cultura, procesos y tec- } \\
\text { nología }\end{array}$ \\
\hline
\end{tabular}

Fuente: elaboración propia a partir de la revisión de literatura. 


\section{Síntesis de los MMGC}

La revisión de literatura en materia de MMGC realizada a través de bases de datos académicas, en regiones como América del Norte, Europa, Asia, África y América Latina (incluyendo Colombia), proporcionó un total de 24 modelos, los cuales tie- nen entre tres (3) y seis (6) niveles de madurez y diversas áreas clave. En la mayoría de modelos se encuentran presentes las áreas clave relacionadas con las personas, la cultura organizacional, los procesos y las tecnologías que se emplean para gestionar el conocimiento organizacional. En la tabla 3 se presenta una relación de dichos modelos.

Tabla 3. Modelos para medir la madurez de GC encontrados en el estado del arte

\begin{tabular}{|c|c|c|}
\hline Modelo & Autor(es) & Año \\
\hline Knowledge Process Quality Model (KPQM) & Paulzen, Doumi, Perc \& Cereijo-Roibas & 2002 \\
\hline Organizational Self Assessment of Knowledge Management Maturity & Kulkarni \& St Louis & 2003 \\
\hline $\begin{array}{l}\text { Diagnóstico de la gestión de conocimiento en una empresa grande de Barranquilla (Co- } \\
\text { lombia) }\end{array}$ & González, Castro \& Roncallo & 2004 \\
\hline Strategic knowledge management maturity model & Snyman \& Kruger & 2005 \\
\hline A Knowledge Management Maturity Model and Application & Feng & 2006 \\
\hline $\begin{array}{l}\text { Gartner: A Knowledge Management Maturity Model Explains Where You 're Going and How } \\
\text { to Get There }\end{array}$ & Harris & 2006 \\
\hline Modelo general de madurez de GC (G-KMMM) & Teah, Pee \& Kankanhalli & 2006 \\
\hline The Support Center Maturity Model & Joslin & 2007 \\
\hline Knowledge management profile maturity model & $\begin{array}{l}\text { Gaál, Szabó, Kovács, Obermayer-Kovács \& } \\
\text { Csepregi }\end{array}$ & 2008 \\
\hline knowledge navigator model (KNM) & Hsieh, Lin \& Lin & 2009 \\
\hline Karagabi KM Model & González, Joaquí \& Collazos & 2009 \\
\hline $\begin{array}{l}\text { Estudios de caso sobre la GC en cuatro organizaciones colombianas líderes en penetración } \\
\text { de mercado }\end{array}$ & Briceño \& Bernal & 2010 \\
\hline Facilitating new knowledge creation and obtaining KM maturity & Arling \& Chun & 2011 \\
\hline Knowledge Management Maturity Model : An Engineering Approach & Kuriakose, Raj, Satya Murty \& Swaminathan & 2011 \\
\hline KM3 & Oliveira, Pedron, Romão \& Becker & 2011 \\
\hline $\begin{array}{l}\text { Análisis de madurez de la gestión del capital intelectual en la pequeña y mediana empresa } \\
\text { latinoamericana }\end{array}$ & Romero \& Pascual & 2011 \\
\hline La GC en Instituciones de Educación Superior (IES) de Medellín & Uribe & 2013 \\
\hline $\begin{array}{l}\text { Análisis comparativo de los modelos de madurez de la GC en grandes empresas de Me- } \\
\text { dellín-Colombia }\end{array}$ & Durango \& Pérez & 2013 \\
\hline Metodología para evaluar la madurez de la GC en algunas grandes empresas colombianas & Durango, Quintero \& Ruiz & 2013 \\
\hline $\begin{array}{l}\text { Madurez de la GC y la gestión de procesos en empresas que utilizan la plataforma virtual } \\
\text { TALENTUM }\end{array}$ & Acuña, Gómez, \& Pintor & 2014 \\
\hline Knowledge Management Maturity in Construction Companies & Wibowo \& Waluyo & 2015 \\
\hline Knowledge Management Maturity Level in a Brazilian Air Force Flight Test Environment & Follador & 2015 \\
\hline Exploring knowledge management maturity from funcionalist and interpretivist perspectives. & Arias-Pérez \& Durango-Yepes & 2015 \\
\hline $\begin{array}{l}\text { Construcción De Un MMGC Para Una Multinacional De Alimentos De Una Economía Emer- } \\
\text { gente. }\end{array}$ & $\begin{array}{l}\text { Arias-Pérez, Tavera-Mesías \& Castaño- } \\
\text { Serna }\end{array}$ & 2016 \\
\hline
\end{tabular}

Fuente: elaboración propia a partir de la revisión de literatura. 
En este apartado se ha realizado una síntesis de los diferentes trabajos que han propuesto, analizado o utilizado los MMGC.

\section{Resultados y discusión}

El análisis comparativo de los MMGC aplicando los criterios de comparación propuestos, arrojó los siguientes resultados:

- Criterio de niveles de madurez. En términos generales, los modelos presentan entre 4 y 6 niveles de madurez, a diferencia de los modelos presentados en los artículos denominados $\mathrm{Fa}$ cilitating new knowledge creation and obtaining KM maturity y Knowledge Management Maturity Level in a Brazilian Air Force Flight Test Environment, los cuales proponen únicamente 3 niveles de madurez. Los modelos presentados en los artículos de investigación denominados Knowledge management profile maturity model, Karagabi KM Model, Estudios de caso sobre la GC en cuatro organizaciones colombianas líderes en penetración de mercado y La GC en Instituciones de Educación Superior (IES) de Medellín no definen niveles de madurez. Los niveles de madurez propuestos en los modelos analizados son similares en características; parten de un nivel inicial (donde la organización tiene una concepción básica de la GC) hasta llegar a los niveles más altos (donde la organización lleva un proceso de mejoramiento continuo y optimización en cuanto a procesos de GC).

- Criterio de áreas o elementos clave. Los modelos en general, proponen entre 3 y 5 áreas clave, a excepción de los modelos presentados en los artículos denominados Knowledge $\mathrm{Ma}$ nagement Maturity in Construction Companies (que no define áreas clave), Análisis comparativo de los modelos de madurez de la GC en grandes empresas de MedellínColombia (que define 2 áreas clave), Diagnóstico de la gestión de conocimiento en una empresa grande de Barranquilla (que define 6 variables), organizaciones colombianas líderes en penetración de mercado (que define 6 áreas clave) y Knowledge management profile maturity model (que define 7 áreas clave). Las áreas clave propuestas por los modelos difieren en su denominación, no obstante, la gran mayoría de los modelos tienen en común el hecho de contar con áreas clave relacionadas con las personas, la cultura organizacional, los procesos y las tecnologías que se utilizan para gestionar el conocimiento organizacional.

- Criterio Instrumento. Dentro de los modelos encontrados en la revisión de literatura, los únicos que no proponen un instrumento para aplicar a los colaboradores de la organización para la medición de la madurez de la GC, son los presentados en los estudios denominados $\mathrm{Ka}$ ragabi KM Model y Facilitating new knowledge creation and obtaining KM maturity. Estos modelos proponen principalmente la realización de entrevistas a directivos (con preguntas abiertas) y la revisión de fuentes secundarias y de los procedimientos para identificar el estado en el que se encuentra la organización en materia de GC.

- Criterio brevedad del instrumento. Para la identificación de este criterio se revisó que el instrumento fuera de fácil comprensión, es decir, que no empleara un lenguaje demasiado técnico y todos los colaboradores de la organización lo pudiesen diligenciar. Adicionalmente, se revisó que el instrumento no tuviese un número muy elevado de preguntas para que los colaboradores de la organización no se rehúsen a diligenciarlo. En este sentido, se definió que los instrumentos que en este criterio cuentan con una valoración alta, serían aquellos que emplean un vocabulario común y de fácil entendimiento y que además, tengan un número adecuado de preguntas (inferior a 50). Los instrumentos que cuentan con la valoración media son aquellos que de igual forma cuentan con un vocabulario de fácil entendimiento pero que tienen más de 50 preguntas.

En términos generales, los instrumentos de los modelos encontrados tienen una valoración entre media y alta en este criterio, ninguno tiene la valoración baja, 
por lo cual es posible inferir que pueden ser empleados en cualquier organización sin la necesidad de realizar algún tipo de capacitación previa a su aplicación. No obstante, dentro de la revisión de literatura no se encontró información sobre el instrumento que aplican los modelos propuestos en los siguientes artículos de investigación: Diagnóstico de la gestión de conocimiento en una empresa grande de Barranquilla (Colombia), Strategic knowledge management maturity model, A Knowledge Management Maturity Model and Application y Gartner: A Knowledge Management Maturity Model Explains Where You're Going and How to Get There.

- Criterio idioma. La gran mayoría de modelos encontrados en la revisión de literatura se encuentran disponibles en inglés o español, lo cual facilita su aplicación. El único modelo que se encuentra en un idioma distinto es el KM3, el cual se encuentra en portugués.

- Criterio complejidad. Los modelos evaluados bajo este criterio se encuentran en un nivel de complejidad entre bajo y medio, esto quiere decir que son flexibles y prácticos de aplicar debido a que proponen una metodología clara y cuentan con instrumentos que tienen criterios de evaluación bien definidos. Los modelos con una valoración de baja en este criterio son aquellos que son más fáciles en su aplicación que aquellos con la valoración media, estos modelos son: A Knowledge Management Maturity Model and Application, Modelo general de madurez de GC (G-KMMM), Análisis comparativo de los modelos de madurez de la GC en grandes empresas de Medellín-Colombia, Metodología para evaluar la madurez de la GC en algunas grandes empresas colombianas, Exploring knowledge management maturity from funcionalist and interpretivist perspectives y Construcción De Un MMGC Para Una Multinacional De Alimentos De Una Economía Emergente.

- Criterio amplio y adaptable. La mayoría de los modelos encontrados en la revisión de literatura cuentan con una amplia documentación y facilidad de adaptación a diferentes contextos organizacionales, a excepción de los modelos Karagabi KM Model, KM3 y Análisis de madurez de la gestión del capital intelectual en la pequeña y mediana empresa latinoamericana.

- Criterio libre uso. De los 24 modelos encontrados en la revisión de literatura, sólo los modelos propuestos en los artículos de investigación denominados Organizational Self Assessment of Knowledge Management Maturity y Modelo general de madurez de GC (G-KMMM) se pueden utilizar libremente en su totalidad, ya que brindan toda la metodología para la medición de la madurez de GC organizacional y proporcionan el instrumento para dicha medición. En la tabla 4 se presentan los 24 MMGC encontrados y los criterios de comparación asociados.

Tabla 4. Criterios de comparación aplicados a los MMGC

\begin{tabular}{|l|l|l|l|l|l|l|l|}
\hline \multicolumn{1}{|c|}{ Modelo } & $\begin{array}{c}\text { Niveles de } \\
\text { madurez }\end{array}$ & $\begin{array}{c}\text { Áreas o } \\
\text { elementos } \\
\text { clave }\end{array}$ & Instrumento & $\begin{array}{c}\text { Brevedad } \\
\text { del instru- } \\
\text { mento }\end{array}$ & $\begin{array}{l}\text { Idioma } \\
\text { Ildad }\end{array}$ & $\begin{array}{c}\text { Comple- } \\
\text { jidadaptable } \\
\text { adas }\end{array}$ & $\begin{array}{l}\text { Libre } \\
\text { uso }\end{array}$ \\
\hline $\begin{array}{l}\text { Knowledge Process Quality Model } \\
\text { (KPQM) }\end{array}$ & 5 etapas & 4 niveles & Sí & Media & Inglés & Media & Sí \\
\hline $\begin{array}{l}\text { Organizational Self Assessment of } \\
\text { Knowledge Management Maturity }\end{array}$ & 5 niveles & 4 áreas clave & Sí & Media & Inglés & Media & Sí \\
\hline $\begin{array}{l}\text { Diagnóstico de la gestión de cono- } \\
\text { cimiento en una empresa grande } \\
\text { de Barranquilla (Colombia) }\end{array}$ & 5 niveles & $\begin{array}{l}6 \text { variables } \\
\text { macro clave }\end{array}$ & Sí & Desconocido & Español & Media & Sí \\
\hline $\begin{array}{l}\text { Strategic knowledge management } \\
\text { maturity model }\end{array}$ & 4 niveles & 4 áreas clave & Sí & Desconocido & Inglés & Media & Sí \\
\hline
\end{tabular}




\begin{tabular}{|c|c|c|c|c|c|c|c|c|}
\hline Modelo & $\begin{array}{c}\text { Niveles de } \\
\text { madurez }\end{array}$ & $\begin{array}{l}\text { Áreas o } \\
\text { elementos } \\
\text { clave }\end{array}$ & Instrumento & $\begin{array}{l}\text { Brevedad } \\
\text { del instru- } \\
\text { mento }\end{array}$ & Idioma & $\begin{array}{l}\text { Comple- } \\
\text { jidad }\end{array}$ & $\begin{array}{c}\text { Amplio y } \\
\text { adaptable }\end{array}$ & $\begin{array}{c}\text { Libre } \\
\text { uso }\end{array}$ \\
\hline $\begin{array}{l}\text { A Knowledge Management Maturi- } \\
\text { ty Model and Application }\end{array}$ & 5 niveles & 3 áreas clave & Sí & Desconocido & Inglés & Baja & Sí & No \\
\hline $\begin{array}{l}\text { Gartner: A Knowledge Manage- } \\
\text { ment Maturity Model Explains } \\
\text { Where You ' re Going and How to } \\
\text { Get There }\end{array}$ & 6 niveles & 3 áreas clave & Sí & Desconocido & Inglés & Media & Sí & No \\
\hline $\begin{array}{l}\text { Modelo general de madurez de GC } \\
\text { (G-KMMM) }\end{array}$ & 5 niveles & 3 áreas clave & Sí & Alta & Inglés & Baja & Sí & Sí \\
\hline The Support Center Maturity Model & 4 fases & 4 áreas clave & Sí & Media & Inglés & Media & Sí & No \\
\hline $\begin{array}{l}\text { Knowledge management profile } \\
\text { maturity model }\end{array}$ & No define & 7 áreas clave & Sí & Media & Inglés & Media & Sí & No \\
\hline knowledge navigator model (KNM) & 5 etapas & 3 áreas clave & Sí & Media & Inglés & Media & Sí & No \\
\hline Karagabi KM Model & No define & 3 áreas clave & No & No aplica & Español & Alta & No & No \\
\hline $\begin{array}{l}\text { Estudios de caso sobre la GC en } \\
\text { cuatro organizaciones colom- } \\
\text { bianas líderes en penetración de } \\
\text { mercado }\end{array}$ & No define & 6 áreas clave & Sí & Media & Español & Media & Sí & No \\
\hline $\begin{array}{l}\text { Facilitating new knowledge crea- } \\
\text { tion and obtaining KM maturity }\end{array}$ & 3 niveles & $\begin{array}{l}4 \text { elementos } \\
\text { clave }\end{array}$ & No & No aplica & Inglés & Media & Sí & No \\
\hline $\begin{array}{l}\text { Knowledge Management Maturity } \\
\text { Model : An Engineering Approach }\end{array}$ & 6 niveles & 5 áreas clave & Sí & Media & Inglés & Media & Sí & No \\
\hline KM3 & 4 niveles & 3 áreas clave & Sí & Media & Portugués & Media & No & No \\
\hline $\begin{array}{l}\text { Análisis de madurez de la gestión } \\
\text { del capital intelectual en la pequeña } \\
\text { y mediana empresa latinoameri- } \\
\text { cana }\end{array}$ & 4 niveles & 3 áreas clave & No & No aplica & Español & Alta & No & No \\
\hline $\begin{array}{l}\text { La GC en Instituciones de Educa- } \\
\text { ción Superior (IES) de Medellín }\end{array}$ & No define & 5 áreas clave & Sí & Desconocido & Español & Media & Sí & No \\
\hline $\begin{array}{l}\text { Análisis comparativo de los mo- } \\
\text { delos de madurez de la GC en } \\
\text { grandes empresas de Medellín- } \\
\text { Colombia }\end{array}$ & 5 niveles & 2 áreas clave & Sí & Alta & Español & Baja & Sí & No \\
\hline $\begin{array}{l}\text { Metodología para evaluar la madu- } \\
\text { rez de la GC en algunas grandes } \\
\text { empresas colombianas }\end{array}$ & 5 niveles & 4 áreas clave & Sí & Alta & Español & Baja & Sí & No \\
\hline $\begin{array}{l}\text { Madurez de la GC y la gestión de } \\
\text { procesos en empresas que utilizan } \\
\text { la plataforma virtual TALENTUM }\end{array}$ & 5 niveles & $\begin{array}{ll}5 & \text { elementos } \\
\text { clave }\end{array}$ & Sí & Media & Español & Media & Sí & No \\
\hline $\begin{array}{l}\text { Knowledge Management Maturity } \\
\text { in Construction Companies }\end{array}$ & 4 niveles & No define & Sí & Media & Inglés & Media & Sí & No \\
\hline $\begin{array}{l}\text { Knowledge Management Maturity } \\
\text { Level in a Brazilian Air Force Flight } \\
\text { Test Environment }\end{array}$ & 3 niveles & 4 áreas clave & Sí & Alta & Inglés & Media & Sí & No \\
\hline $\begin{array}{l}\text { Exploring knowledge management } \\
\text { maturity from funcionalist and in- } \\
\text { terpretivist perspectives. }\end{array}$ & 5 niveles & 4 áreas clave & Sí & Alta & Inglés & Baja & Sí & No \\
\hline $\begin{array}{l}\text { Construcción De Un MMGC Para } \\
\text { Una Multinacional De Alimentos De } \\
\text { Una Economía Emergente. }\end{array}$ & 5 niveles & 4 áreas clave & Sí & Alta & Español & Baja & Sí & No \\
\hline
\end{tabular}

Fuente: elaboración propia a partir de la revisión de literatura. 
Una vez definida la escala de evaluación para cada uno de los criterios de comparación, se procedió a la evaluación cuantitativa de los modelos, lo cual se encuentra consignando en la tabla 5.

Tabla 5. Evaluación cuantitativa para la selección del modelo

\begin{tabular}{|c|c|c|c|c|c|c|c|c|c|}
\hline Modelo & $\begin{array}{c}\text { Niveles de } \\
\text { madurez }\end{array}$ & $\begin{array}{l}\text { Áreas o } \\
\text { elementos } \\
\text { clave }\end{array}$ & Instrumento & $\begin{array}{l}\text { Brevedad del } \\
\text { instrumento }\end{array}$ & Idioma & $\begin{array}{c}\text { Compleji- } \\
\text { dad }\end{array}$ & $\begin{array}{c}\text { Amplio y } \\
\text { adaptable }\end{array}$ & $\begin{array}{c}\text { Libre } \\
\text { uso }\end{array}$ & $\begin{array}{l}\text { Califica- } \\
\text { ción }\end{array}$ \\
\hline $\begin{array}{l}\text { Modelo general de madurez } \\
\text { de GC (G-KMMM) }\end{array}$ & 1 & 0,5 & 1 & 1 & 0,5 & 1 & 1 & 1 & 7 \\
\hline $\begin{array}{l}\text { Análisis comparativo de los } \\
\text { modelos de madurez de la } \\
\text { GC en grandes empresas } \\
\text { de Medellín-Colombia }\end{array}$ & 1 & 0,5 & 1 & 1 & 1 & 1 & 1 & 0 & 6,5 \\
\hline $\begin{array}{l}\text { Metodología para evaluar } \\
\text { la madurez de la GC en } \\
\text { algunas grandes empresas } \\
\text { colombianas }\end{array}$ & 1 & 0,5 & 1 & 1 & 1 & 1 & 1 & 0 & 6,5 \\
\hline $\begin{array}{l}\text { Construcción De Un MMGC } \\
\text { Para Una Multinacional De } \\
\text { Alimentos De Una Econo- } \\
\text { mía Emergente. }\end{array}$ & 1 & 0,5 & 1 & 1 & 1 & 1 & 1 & 0 & 6,5 \\
\hline $\begin{array}{l}\text { Organizational Self As- } \\
\text { sessment of Knowledge } \\
\text { Management Maturity }\end{array}$ & 1 & 0,5 & 1 & 0,5 & 0,5 & 0,5 & 1 & 1 & 6 \\
\hline $\begin{array}{l}\text { Exploring knowledge mana- } \\
\text { gement maturity from fun- } \\
\text { cionalist and interpretivist } \\
\text { perspectives*. }\end{array}$ & 1 & 0,5 & 1 & 1 & 0,5 & 1 & 1 & 0 & 6 \\
\hline $\begin{array}{l}\text { Diagnóstico de la gestión } \\
\text { de conocimiento en una } \\
\text { empresa grande de Barran- } \\
\text { quilla (Colombia) }\end{array}$ & 1 & 1 & 1 & 0 & 1 & 0,5 & 1 & 0 & 5,5 \\
\hline $\begin{array}{l}\text { Madurez de la GC y la ges- } \\
\text { tión de procesos en empre- } \\
\text { sas que utilizan la platafor- } \\
\text { ma virtual TALENTUM }\end{array}$ & 1 & 0,5 & 1 & 0,5 & 1 & 0,5 & 1 & 0 & 5,5 \\
\hline $\begin{array}{l}\text { Knowledge Process Quality } \\
\text { Model (KPQM) }\end{array}$ & 1 & 0,5 & 1 & 0,5 & 0,5 & 0,5 & 1 & 0 & 5 \\
\hline $\begin{array}{l}\text { A Knowledge Management } \\
\text { Maturity Model and Appli- } \\
\text { cation }\end{array}$ & 1 & 0,5 & 1 & 0 & 0,5 & 1 & 1 & 0 & 5 \\
\hline $\begin{array}{l}\text { The Support Center Maturi- } \\
\text { ty Model }\end{array}$ & 1 & 0,5 & 1 & 0,5 & 0,5 & 0,5 & 1 & 0 & 5 \\
\hline $\begin{array}{l}\text { knowledge navigator model } \\
\text { (KNM) }\end{array}$ & 1 & 0,5 & 1 & 0,5 & 0,5 & 0,5 & 1 & 0 & 5 \\
\hline $\begin{array}{l}\text { Estudios de caso sobre la } \\
\text { GC en cuatro organizacio- } \\
\text { nes colombianas líderes en } \\
\text { penetración de mercado }\end{array}$ & 0 & 1 & 1 & 0,5 & 1 & 0,5 & 1 & 0 & 5 \\
\hline
\end{tabular}




\begin{tabular}{|c|c|c|c|c|c|c|c|c|c|}
\hline Modelo & $\begin{array}{c}\text { Niveles de } \\
\text { madurez }\end{array}$ & $\begin{array}{l}\text { Áreas o } \\
\text { elementos } \\
\text { clave }\end{array}$ & Instrumento & $\begin{array}{l}\text { Brevedad del } \\
\text { instrumento }\end{array}$ & Idioma & $\begin{array}{c}\text { Compleji- } \\
\text { dad }\end{array}$ & $\begin{array}{c}\text { Amplio y } \\
\text { adaptable }\end{array}$ & \begin{tabular}{|c} 
Libre \\
uso
\end{tabular} & $\begin{array}{l}\text { Califica- } \\
\text { ción }\end{array}$ \\
\hline \begin{tabular}{|l|} 
Knowledge Management \\
Maturity Model: An Engi- \\
neering Approach \\
\end{tabular} & 1 & 0,5 & 1 & 0,5 & 0,5 & 0,5 & 1 & 0 & 5 \\
\hline $\begin{array}{l}\text { Knowledge Management } \\
\text { Maturity Level in a Brazilian } \\
\text { Air Force Flight Test Envi- } \\
\text { ronment }\end{array}$ & 0,5 & 0,5 & 1 & 1 & 0,5 & 0,5 & 1 & 0 & 5 \\
\hline $\begin{array}{l}\text { Strategic knowledge mana- } \\
\text { gement maturity model }\end{array}$ & 1 & 0,5 & 1 & 0 & 0,5 & 0,5 & 1 & 0 & 4,5 \\
\hline $\begin{array}{l}\text { Gartner: A Knowledge } \\
\text { Management Maturity Mo- } \\
\text { del Explains Where You ' } \\
\text { re Going and How to Get } \\
\text { There }\end{array}$ & 1 & 0,5 & 1 & 0 & 0,5 & 0,5 & 1 & 0 & 4,5 \\
\hline \begin{tabular}{|l|} 
Knowledge management \\
profile maturity model
\end{tabular} & 0 & 1 & 1 & 0,5 & 0,5 & 0,5 & 1 & 0 & 4,5 \\
\hline \begin{tabular}{|l|} 
Knowledge Management \\
Maturity in Construction \\
Companies
\end{tabular} & 1 & 0 & 1 & 0,5 & 0,5 & 0,5 & 1 & 0 & 4,5 \\
\hline $\begin{array}{l}\text { La GC en Instituciones de } \\
\text { Educación Superior (IES) } \\
\text { de Medellin }\end{array}$ & 0 & 0,5 & 1 & 0 & 1 & 0,5 & 1 & 0 & 4 \\
\hline KM3 & 1 & 0,5 & 1 & 0,5 & 0 & 0,5 & 0 & 0 & 3,5 \\
\hline $\begin{array}{l}\text { Facilitating new knowledge } \\
\text { creation and obtaining KM } \\
\text { maturity }\end{array}$ & 0,5 & 0,5 & 0 & 0 & 0,5 & 0,5 & 1 & 0 & 3 \\
\hline $\begin{array}{l}\text { Análisis de madurez de } \\
\text { la gestión del capital in- } \\
\text { telectual en la pequeña y } \\
\text { mediana empresa latinoa- } \\
\text { mericana }\end{array}$ & 1 & 0,5 & 0 & 0 & 1 & 0 & 0 & 0 & 2,5 \\
\hline Karagabi KM Model & 0 & 0,5 & 0 & 0 & 1 & 0 & 0 & 0 & 1,5 \\
\hline
\end{tabular}

Fuente: elaboración propia a partir de la revisión de literatura.

De los modelos encontrados, se identificó que el MMGC con mayor calificación fue el denominado Modelo general de madurez de GC (G-KMMM) de Teah, Pee \& Kankanhalli (2006), el cual tuvo una puntuación de 7. Esto significa que es el modelo más integral, con mayores fortalezas y mejores características para la realización del diagnóstico de madurez de GC, de los analizados.

Una de las fortalezas más representativas del modelo G-KMMM, es que se puede aplicar a diferentes objetos de análisis, incluidos la organización como conjunto y de manera individual para sus respectivas unidades. Adicionalmente, el modelo brinda una explicación muy detallada del instrumento de medición para proporcionar un enfoque sistemático y estructurado que garantiza la transparencia del procedimiento de evaluación. Es importante señalar que el modelo G-KMMM adopta una estructura organizada y define claramente cada nivel de madurez y sus áreas clave, así como sus respectivas características (Teah et al., 2006). 


\section{Consideraciones finales}

En este trabajo además de realizar una síntesis de los diferentes trabajos que han propuesto, analizado o utilizado los MMGC, se elaboró un análisis comparativo de los mismos a partir de los criterios propuestos por Martínez (2015), con el fin de evaluar la usabilidad de un MMGC.

De la revisión de la literatura se identificó que si bien los MMGC se derivan de los modelos de madurez del área de ingeniería y sistemas, la aplicación específica al área de gestión de conocimiento se da sólo a partir de 2001 .

De los 24 modelos encontrados entre 2001 y 2016 , se estableció que el MMGC con mayor calificación fue el denominado Modelo general de madurez de GC (G-KMMM) de Teah, Pee \& Kankanhalli (2006). Esto significa que es el modelo más integral, con mayores fortalezas y mejores características para la realización del diagnóstico de madurez de GC, entre los analizados. No obstante, dado que este modelo es de 2006, es posible que se puedan realizar adaptaciones del mismo, con el fin de actualizarlo y garantizar una medición más robusta de la madurez de GC en una organización.

Entre los trabajos futuros de investigación se encuentra: la construcción de una versión adaptada del MMGC de Teah, Pee \& Kankanhalli (2006); la aplicación a organizaciones no tradicionales, posiblemente en el sector educativo o de servicios.

\section{Referencias}

Acuña, J., Gómez, M., \& Pintor, M. (2014). Madurez de la gestión del conocimiento y la gestión de procesos en empresas que utilizan la plataforma virtual TALENTUM. Ciencias de La Información, 45 (2): 49-55.

Arias, J. (2012). Asociaciones entre madurez de gestión del conocimiento y desempeño innovador: Organización y personas, e interpretación. Revista Lasallista de Investigación, 9 (1): 86-95.

Arias, J., \& Tavera, J. (2015). Linking knowledge management maturity and innovation in leading companies in research and development. Revista Republicana, 18: 159-180.

Arias-Pérez, J., \& Durango-Yepes, C. (2015). Exploring knowledge management maturity from funcionalist and interpretivist perspectives. Entramado, 11 (1):
94-104. http://doi.org/https://dx.doi.org/10.18041/ entramado. $2015 \mathrm{v} 11 \mathrm{n} 1.21112$

Arias-Pérez, J., Tavera-Mesías, J., \& Castaño-Serna, D. (2016). Construcción de un modelo de madurez de gestión del conocimiento para una multinacional de alimentos de una economía emergente. El Profesional de La Información, 25 (1): 88-102. http://doi.org/http://dx.doi.org/10.3145/epi.2016.ene.09

Arling, P., \& Chun, M. (2011). Facilitating new knowledge creation and obtaining KM maturity. Journal of Knowledge Management, 15 (2): 231-250. http://doi. org/10.1108/13673271111119673

Briceño, M. \& Bernal, C. (2010). Estudios de caso sobre la gestión del conocimiento en cuatro organizaciones colombianas líderes en penetración de mercado. Estudios Gerenciales, 26 (117): 173-193. http://doi.org/10.1016/S0123-5923(10)70140-6

Durango, C., \& Pérez, J. (2013). Análisis comparativo de los modelos de madurez de la gestión del conocimiento en grandes empresas de Medellín-Colombia. Biblioteca Digital de La Asociación Latino-Iberoamericana de Gestión Tecnológica, 1 (1). Retrieved from http://www.altec-dl.org/index.php/ altec/article/view/1948

Durango, C., Quintero, M., \& Ruiz, C. (2013). Metodología para evaluar la madurez de la gestión del conocimiento en algunas grandes empresas colombianas. Tecnura, 19 (43): 20-36. http://doi.org/http://dx.doi.org/10.14483/udistrital.jour. tecnura.2015.1.a01

Feng, J. (2006). A knowledge management maturity model and application. Paper Presented at the Technology Management for the Global Future, 2006. PICMET 2006.

Follador, R. C. (2015). Knowledge Management Maturity Level in a Brazilian Air Force Flight Test Environment. 2015 Portland International Conference on Management of Engineering and Technology (PICMET), 1296-1304. http://doi.org/ http://dx.doi.org/10.1109/PICMET.2015.7272952

Gaál, Z., Szabó, L., Kovács, Z., Obermayer-Kovács, N., \& Csepregi, A. (2008). Knowledge management profile maturity model. Paper Presented at the Proceedings of 9th European Conference on Knowledge Management (ECKM 2008), Southampton Solent University, Southampton, UK.

González, A., Castro, J., \& Roncallo, M. (2004). Diagnóstico de la gestión de conocimiento en una empresa grande de Barranquilla (Colombia): Una actividad de vinculación cooperativa universidad - sector productivo. Ingeniería Y Desarrollo: Revista de La División de Ingeniería de La Universidad Del Norte, (16): 70-103. Retrieved from http://dialnet. unirioja.es/servlet/articulo?codigo $=2508538 \&$ info=resume n\&idioma $=\mathrm{ENG}$

González, A., Joaquí, C., \& Collazos, C. (2009). Karagabi Kmmodel: modelo de referencia para la introducción de iniciativas de gestión del conocimiento en organizaciones basadas en conocimiento. Ingeniare. Revista Chilena de Ingeniería, 17 (2): $224-235$.

Harris, K. (2006). Gartner A Knowledge Management Maturity Model Explains Where You 're Going and How to Get There, (February).

Heinzen, A. (2015). Avaliação da maturidade da gestão do conhecimento na administração pública. Universidade 
Federal de Santa Catarina. Retrieved from http://btd.egc. ufsc.br/wp-content/uploads/2015/06/Angela-Regina-Heinzen-Amin-Helou.pdf

Hsieh, P. J., Lin, B., \& Lin, C. (2009). The construction and application of knowledge navigator model (KNM): An evaluation of knowledge management maturity. Expert Systems with Applications, 36 (2 PART 2): 4087-4100. http://doi. org/10.1016/j.eswa.2008.03.005

Joslin, R. (2007). The Knowledge Management Maturity Model. CRM Magazine, 11 (11): 8-18.

Klimko, G. (2001). Knowledge management and maturity models: Building common understanding. Proceeding of the $2 \mathrm{nd} \mathrm{Eu-}$ ropean Conference on Knowledge Management, 269-278.

Kulkarni, U., \& St Louis, R. (2003). Organizational self assessment of knowledge management maturity. AMCIS 2003 Proceedings, 332.

Kuriakose, K., Raj, B., Satya Murty, S., \& Swaminathan, P. (2010). Knowledge management maturity models-a morphological analysis. J Knowl Manag Pract, 11 (3).

Kuriakose, K., Raj, B., Satya Murty, S., \& Swaminathan, P. (2011). Knowledge Management Maturity Model: An Engineering Approach. Journal Of Knowledge Management Practice, 12 (2): 1-14.

Martínez, J. (2015). Modelo de madurez en el dominio de los proyectos aplicado a organizaciones de Gestión de Proyectos en Medellín. Universidad EAFIT. Retrieved from https://repository.eafit.edu.co/xmlui/bitstream/handle/10784/7291/Jeferson_MartinezLozano_2015_Principal. pdf?sequence $=2 \&$ isAllowed $=y$

Nonaka, I., \& Takeuchi, H. (1995). The knowledge-creating company: How Japanese companies create the dynamics of innovation. Oxford university press.

Olarte-Reyes, M. \& Lis-Gutiérrez, J. (2015) Gestión de activos intelectuales y creación de valor. Administración y Desarrollo, [S.1.], 45 (2): 190-207. ISSN 2500-5227. Disponible en: <http://esapvirtual.esap.edu.co/ojs/index.php/admindesarro/ article/view/11>. Fecha de acceso: 13 ene. 2017 doi:http:// dx.doi.org/10.22431/25005227.11.

Oliveira, M., Pedron, C., Romao, M., \& Becker, G. (2011). Proposta de um modelo de maturidade para Gestão do Conhecimento: KM3. Revista Portuguesa E Brasileira de Gestao, 10 (4): 11-25.

Paulk, M., Curtis, B., Chrissis, M., \& Weber, C. (1993). Capability maturity model, version 1.1. Software, IEEE, 10 (4): 18-27.

Paulzen, O., Doumi, M., Perc, P., \& Cereijo-Roibas, A. (2002). A Maturity Model for Quality Improvement in Knowledge Management. ACIS 2002 Proceedings, 5: 1-12.

Prieto, R., Meneses, C., \& Vega, V. (2015). Análisis comparativo de modelos de madurez en inteligencia de negocio. Ingeniare. Revista Chilena de Ingeniería, 23 (3): 361-371. Retrieved from http://www.scielo.cl/pdf/ingeniare/v23n3/art05.pdf

Romero, D., \& Pascual, F. (2011). Análisis de madurez de la gestión del capital intelectual en la pequeña y mediana empresa latinoamericana. Revista Da Micro E Pequeña Empresa, 5 (3), 40-60.

Serna, E. (2012). Maturity model of knowledge management in the interpretativist perspective. International Journal of Information Management, 32 (4): 365-371. http://doi. org/10.1016/j.ijinfomgt.2011.12.001

Snyman, M., \& Kruger, C. (2005). Formulation of a strategic knowledge management maturity model. South African Journal of Information Management, 7 (2).

Teah, H. Y., Pee, L. G., \& Kankanhalli, A. (2006). Development and application of a general knowledge management maturity model. The Tenth Pacific Asia Conference on Information Systems (PACIS 2006), (Pacis), 401-416.

Uribe, A. (2013). La Gestión del conocimiento en Instituciones de Educación Superior (IES) de Medellín. Escenarios: Empresa Y Territorio, (2): 243-258.

Wibowo, M. A., \& Waluyo, R. (2015). Knowledge Management Maturity in Construction Companies. Procedia Engineering, 125: 89-94. 